R. Fries

M. Böhm

Tiefe Beinvenenthrombose - Therapie

\title{
Deep venous thrombosis - treatment
}

\section{Therapieziele}

Die Therapie der tiefen Beinvenenthrombose (TVT) zielt in verschiedene Richtungen. Es gilt einerseits die lokale Beschwerdesymptomatik des Patienten zu verbessern, das heißt für ein Abschwellen der betroffenen Extremität zu sorgen und eine schmerzfreie Beweglichkeit wiederherzustellen. Andererseits soll eine potenziell vitale Gefährdung des Patienten durch Eintreten einer Lungenembolie akut und in der Folgezeit verhindert werden. Darüber hinaus soll durch eine möglichst gute Wiederherstellung der Venenfunktion, im Idealfall Rekanalisation mit Erhalt der Klappenfunktion, die Entwicklung eines postthrombotischen Syndroms und seiner Folgekomplikationen verhindert werden.

\section{Erstmaßnahmen}

Nach Sicherung der Diagnose einer TVT sollte umgehend eine effektive Antikoagulation eingeleitet werden. Hierfür kommt die intravenöse Bolusgabe von unfraktioniertem Heparin mit anschließender Dauerinfusion oder die zweimal tägliche subkutane Gabe eines gewichtsadaptierten Bolus eines niedermolekularen Heparins in Frage. Nach intravenöser Bolusinjektion von unfraktioniertem Heparin (üblicherweise 5000 IE) tritt die Wirkung sofort ein. Die anschließende Dauerinfusion mit dem Ziel einer PTT-Verlängerung auf das 1,5-2,5-Fache der Norm erweist sich jedoch in der Praxis als nur schwer steuerbar. Selbst unter Studienbedingungen wird der therapeutische Bereich häufig nicht dauerhaft erreicht. Besser praktikabel ist die Behandlung mit niedermolekularem Heparin subkutan. Der verzögerte Wirkeintritt nach Gabe der ersten Dosis (Erreichen des therapeutischen Bereiches ca. 2-3 Stunden nach Injektion) stellt in der Praxis kein Problem dar. Der Vorteil der Behandlung mit niedermolekularem Heparin liegt in der guten Vorhersagbarkeit des gerinnungshemmenden Effektes, so dass in einem Körpergewichtsbereich zwischen 50 und $100 \mathrm{~kg}$ kein Labormonitoring erforderlich ist. $\mathrm{Zu}$ bedenken ist jedoch dass niedermolekulare Heparine nahezu ausschließlich renal eliminiert werden. Daher beginnen diese Substanzen bereits bei einem Serumkreatinin von $1,5 \mathrm{mg} / \mathrm{dl}$ zu akku- mulieren (Anti-Xa-Spiegelkontrollen ratsam) und sollten ab einem Serumkreatinin von $2 \mathrm{mg} / \mathrm{dl}$ nicht mehr eingesetzt werden. Sofern verfügbar, stellt die Kreatininclearance einen wesentlich genaueren Parameter zur Abschätzung der verbleibenden Nierenfunktion dar. Hier gilt ein Grenzwert von $<30 \mathrm{ml} / \mathrm{min}$ als Schwellenwert. Bei relativer Überdosierung im Rahmen einer Niereninsuffizienz ist insbesondere bei Patienten, die auch mit Thrombozytenaggregationshemmern behandelt werden, mit Blutungskomplikationen zu rechnen. Das Auftreten einer antikörpervermittelten heparininduzierten Thrombozytopenie (HIT) unter der Therapie mit niedermolekularem Heparin ist eine Rarität, wohingegen unter unfraktioniertem Heparin in ca. 3\% mit einer HIT Typ II zu rechnen ist (19). Auch dieser Umstand spricht für die bevorzugte Anwendung niedermolekularer Heparine. Eine Kontrolle der Thrombozytenwerte vor Beginn einer Therapie mit niedermolekularem Heparin, am ersten Tag nach Therapieeinleitung und zweimal wöchentlich bis zum 21. Tag bei Therapiefortführung wird dennoch empfohlen.

In Tab. 1 sind die derzeit zur Behandlung der TVT in Deutschland zugelassenen Substanzen mit den entsprechenden Dosierungsempfehlungen zusammengefasst.

kurzgefasst: Die Therapie der tiefen Beinvenenthrombose soll einerseits die Akutbeschwerden lindern und andererseits potenziell lebensgefährliche Lungenembolien und ein postthrombotisches Syndrom im Langzeitverlauf verhindern. Die subkutane Gabe eines niedermolekularen Heparins in gewichtsadaptierter Dosierung und die Einstellung auf orale Antikoagulation gilt heute als Standardtherapie.

\section{Effektivität von niedermolekularem und unfraktioniertem Heparin}

Die TVT-Therapie mit niedermolekularem Heparin ist der Behandlung mit unfraktioniertem Heparin bezüglich der Endpunkte thromboembolische Komplikationen und Blutungskomplikationen mindestens äquivalent $(8,11,12)$. In einzelnen Studien haben sich die 
Tab.1 Niedermolekulare Heparine zur Behandlung der tiefen Beinvenenthrombose.

\begin{tabular}{|c|c|c|}
\hline \multicolumn{3}{|c|}{ Derzeit zugelassen für die Therapie der TVT in Deutschland: } \\
\hline Substanz & Handelsname & Dosierung \\
\hline Enoxaparin & Clexane $^{\circledR}$ & $2 \times 1 \mathrm{mg} / \mathrm{kg} \mathrm{KG}$ \\
\hline Nadroparin & Fraxiparin $^{\circledR}$ & $2 \times 0,1 \mathrm{ml} / \mathrm{kg} \mathrm{KG}$ \\
\hline Nadroparin & Fraxodi $^{\circledR}$ & $1 \times 0,1 \mathrm{ml} / \mathrm{kg} \mathrm{KG}$ \\
\hline Tinzaparin & Innohep ${ }^{\circledR}$ & $1 \times 175$ I.E./kg KG \\
\hline \multicolumn{3}{|c|}{ Derzeit nicht zugelassen für die Therapie der TVT in Deutschland: } \\
\hline Substanz & Handelsname & Dosierung \\
\hline Certoparin & Mono-Embolex ${ }^{\circledR}$ & $2 \times 8000$ I.E./d \\
\hline Dalteparin & Fragmin $^{\circledR}$ & $2 \times 100$ o. 1 × 200 I.E. $/ \mathrm{kg} \mathrm{KG}$ \\
\hline Reviparin & Clivarin $^{\circledR}$ & $2 \times 87,5$ I.E. $/ \mathrm{kg} \mathrm{KG}$ \\
\hline
\end{tabular}

niedermolekularen Heparine sogar als signifikant überlegen erwiesen. In einer phlebographisch kontrollierten Studie konnte überdies gezeigt werden, dass die Thrombusregression unter einer 7-tägigen Therapie mit niedermolekularem Heparin im Vergleich zu unfraktioniertem Heparin verbessert ist (4). Ob die bessere Thrombusregression auch in eine funktionelle Verbesserung im Langzeitverlauf, also in eine geringere Rate postthrombotischer Syndrome mündet, ist bisher nicht bekannt.

Niedermolekulare Heparine können sowohl mit Einmalgabe der körpergewichtsadaptierten Dosis als auch bei Verteilung auf zwei Tagesdosen sicher und effektiv eingesetzt werden (5). Derzeit kann jedoch nicht ausgeschlossen werden, dass die Einmalgabe mit mehr Blutungskomplikationen einhergeht, was durch den vergleichsweise höheren Wirkgipfel nach Einmalgabe wahrscheinlich erscheint. Bis auf Weiteres sollte bei Patienten mit hohem Blutungsrisiko die zweimal tägliche Gabe bevorzugt werden.

\section{Rezidivprophylaxe}

\section{Rezidivprophylaxe mit oralen Antikoagulantien}

Sofern keine Kontraindikation besteht, kann bereits am Tag der Erstdiagnose mit der Einstellung auf orale Antikoagulation begonnen werden. Die Heparingabe sollte bis zum Erreichen des angestrebten therapeutischen Bereichs (INR 2-3) fortgesetzt werden. Unter Berücksichtigung der besseren Thrombusregression erscheint die mindestens 7-tägige Gabe eines niedermolekularen Heparins empfehlenswert. Bei Patienten mit erhöhtem Blutungsrisiko sollte zur Vermeidung einer Gefährdung durch überlappende effektive orale Antikoagulation und gleichzeitige Heparingabe der Beginn der Einstellung auf orale Antikoagulation verzögert werden.

Die Dauer der Rezidivprophylaxe richtet sich nach dem zu erwartenden Rezidivrisiko. Dieses wird einerseits bestimmt durch die bekannten Risikofaktoren und andererseits durch die Entstehung eines thrombogenen Milieus durch die abheilende Thrombose selbst. Der letztgenannte Faktor ist umso bedeutsamer je ausgedehnter die Thrombose ist. Empfohlen wird eine Rezidivprophylaxe für 3 Monate bei distaler TVT (unterhalb Vena femoralis), für 6 Monate bei proximaler TVT (Oberschenkel und/oder Becken) und für $1 \mathrm{Jahr}$ bei Rezidiv einer TVT oder symptomatischer Lungenembolie. Für eine partielle Unter-

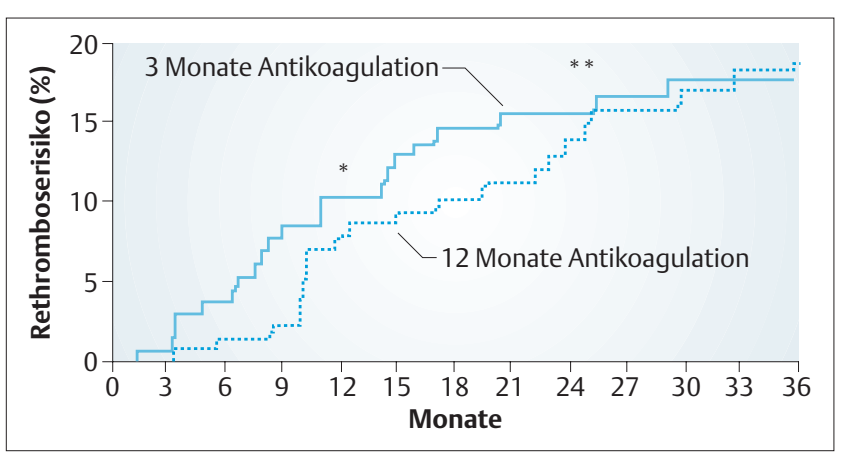

Abb.1 Rethromboserisiko bei 3-monatiger im Vergleich zu 12monatiger Antikoagulation. Nach 12 Monaten $\left({ }^{*}\right)$ trat bei 134 Patienten mit verlängerter Antikoagulation nur in einem Fall eine Rethrombose auf, während von 133 Patienten mit nur 3-monatiger Antikoagulation elf Patienten betroffen waren. Nach 24 Monaten $\left({ }^{* *}\right)$ hatten in beiden Gruppen 21 Patienten eine Rethrombose.

schenkelvenen-TVT (unterhalb der V. poplitea) erscheint in Abhängigkeit von Ausdehnung und duplexsonographischem Verlauf eine 4- bis 6-wöchige Antikoagulation ausreichend. Eine unbefristete Antikoagulation nach TVT ist empfehlenswert bei Beteiligung der Vena cava inferior, bei multiplen Rezidiven, bei einem aktiven Malignom, bei einem Antiphospholipid-Antikörper-Syndrom und bei kombinierten Thrombophilien bzw. einem hereditären AT III-Mangel oder einer homozygoten Faktor V-Leiden-Mutation. Bezüglich des optimalen Verhaltens bei Thrombophilie besteht in vielen Punkten international noch keine Einigkeit.

Im Vergleich einer 3-monatigen Rezidivprophylaxe mit einer 12-monatigen Antikoagulation nach einer ersten TVT unklarer Genese (1) konnte gezeigt werden, dass mit Absetzen der Antikoagulation mit einem signifikanten Wiederanstieg der TVT Rate gerechnet werden muss (Abb.1). Im Zweifel über interkurrente Risiken sollte daher bei fehlenden Kontraindikationen eher großzügiger antikoaguliert werden.

\section{Rezidivprophylaxe mit niedermolekularem Heparin}

Niedermolekulare Heparine sind zur Rezidivprophylaxe nach TVT nicht zugelassen und verursachen im Vergleich mit oralen Antikoagulantien erhebliche Kosten. Sie können jedoch erfolgreich zu diesem Zweck eingesetzt werden. In randomisierten Studien wurde Enoxaparin in einer fixen Dosis von $40 \mathrm{mg} / \mathrm{d}$ und Nadroparin in einer Dosierung von $85 \mathrm{IU} / \mathrm{kg}$ Körpergewicht einmal täglich mit oraler Antikoagulation verglichen $(9,14,18)$. Im Kurzzeitverlauf (3 Monate) erwiesen sich die niedermolekularen Heparine bezüglich der thromboembolischen Komplikationen der oralen Antikoagulation als überlegen bei weniger bzw. vergleichbar vielen Blutungskomplikationen. Unter besonderen Umständen wie z.B. Schwangerschaft oder laufender Chemotherapie können niedermolekulare Heparine also in maximal körpergewichtsadaptierter Dosis einmal täglich alternativ zur oralen Antikoagulation sicher eingesetzt werden. Die Dosisreduktion sollte vorzugsweise nach der ersten Therapiewoche erfolgen. 
kurzgefasst: Niedermolekulare Heparine sind unfraktioniertem Heparin in der Therapie der tiefen Beinvenenthrombose mindestens gleichwertig, wobei die Thrombusregression unter niedermolekularem Heparin ausgeprägter ist. Bei Kontraindikationen gegen eine Rezidivprophylaxe mit oralen Antikoagulantien kann niedermolekulares Heparin in der Hälfte der körpergewichtsadaptierten Tagesdosis alternativ sicher eingesetzt werden. Bei Ausschluss außergewöhnlicher Blutungsrisiken sollte die Rezidivprophylaxe im Zweifelsfall eher länger als kürzer fortgesetzt werden.

\section{Kompressionstherapie}

Die Kompression der betroffenen Extremität soll das Abschwellen des Beines beschleunigen, wirkt in diesem Zusammenhang begrenzt analgetisch und trägt durch Umklammern flottierender Thrombusanteile möglicherweise zur Verhinderung von Lungenembolien bei. Das Bein sollte initial mit elastischen Binden immer über das Thrombusende hinaus komprimiert werden. Bei immobilisierten Patienten werden Langzugbinden empfohlen (hoher Ruhedruck - geringerer Arbeitsdruck), mobilisierte Patienten sollten mit Kurzzugbinden (hoher Belastungsdruck - geringerer Ruhedruck) solange behandelt werden, bis das Bein vollkommen abgeschwollen ist. Anschließend kann ein Kompressionsstrumpf der Klasse II (25-35 mmHg Kompressionsdruck) verordnet werden, wobei die Anpassung eines Strumpfes nach Maß nur ausnahmsweise erforderlich ist. In den meisten Fällen genügt die Verordnung eines knielangen Kompressionsstrumpfes, der unter Berücksichtigung des Tragekomforts auch mit höherer Wahrscheinlichkeit regelmäßig getragen wird. Über Nacht ist der Kompressionsstrumpf wegen der fehlenden hydrostatischen Venenbelastung ebensowenig erforderlich wie routinemäßig am nicht betroffenen Bein. Die Effektivität der Kompressionstherapie zur Verhinderung postthrombotischer Syndrome ist für einen Zeitraum von 2 Jahren belegt (3). Darüber hinaus sollte eine weitere Therapie im Einzelfall vom Funktionszustand des tiefen Leitvenensystems abhängig gemacht werden.

\section{Mobilisation und ambulante Therapie oder Immobilisation im Krankenhaus?}

Die Notwendigkeit strikter Bettruhe nach Diagnose einer TVT ist historisch übermittelt. Diese Tradition wurde lange Zeit nicht hinterfragt und bei Mobilisation ein hohes Lungenembolierisiko unterstellt. Mit der Einführung der niedermolekularen Heparine wurde die ambulante Therapie der TVT möglich und in den entsprechenden Studien zeigte sich keine höhere Embolierate bei den mobilisierten Patienten $(10,13)$. Da die Patienten in diesen Untersuchungen z.T. initial doch stationär aufgenommen wurden und das Ausmaß der Mobilisation kein kontrolliertes Kriterium war, werden die Ergebnisse als Votum für eine frühe Mobilisation und ambulante Therapie nicht allgemein anerkannt. Zudem ist zu bedenken, dass bei Beachtung der wesentlichen Ausschlusskriterien (Tab.2) nur etwa jeder zweite für eine ambulante Therapie überhaupt in Frage kommt. In zwei kleineren randomisierten Studien $(2,16)$ konnte jedoch inzwischen gezeigt werden, dass bei Immobilisation nicht signifikant weniger Lungenembolien auftreten als bei Mobilisation.
Tab.2 Wichtige Ausschlusskriterien für die ambulante Therapie der tiefen Beinvenenthrombose.

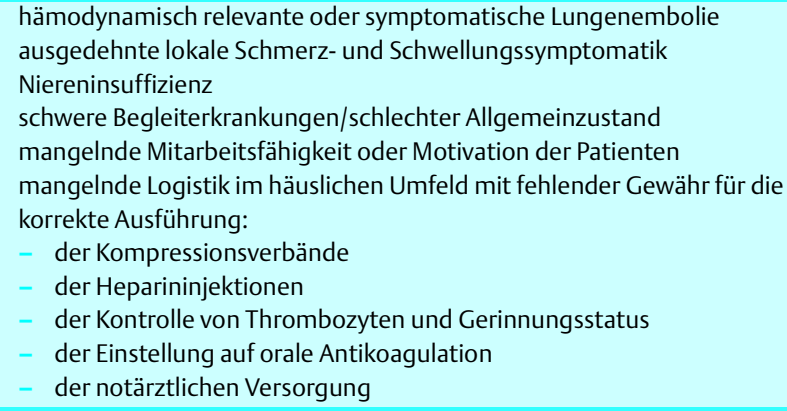

Dies gilt auch für Beckenvenenthrombosen $(2,16)$ und TVT mit flottierendem Thrombusanteil (2). Auch in diesen Untersuchungen erwiesen sich nur weniger als die Hälfte der Patienten als geeignet für eine ambulante Therapie der TVT. Bei optimaler Infrastruktur scheint dieser Anteil jedoch auf nahezu $80 \%$ steigerbar $(15,17)$. Die ambulante Therapie der TVT ist also unter den genannten Voraussetzungen ohne erwiesenen Nachteil für den Patienten durchführbar und im Hinblick auf die bessere Lebensqualität im Vergleich zur stationären Therapie im geeigneten Fall wünschenswert. Die Deutsche Gesellschaft für Angiologie trägt dieser Entwicklung in ihren aktuellen Leitlinien Rechnung und stellt die ambulante Therapie für geeignete Patienten (Tab.2) zur Wahl sofern die folgenden Voraussetzungen erfüllt sind (7):

vollständiges Verständnis des Patienten für die Erkrankung, deren Komplikationen und die vorgesehene Behandlung.

Gewähr, dass die Applikation von Heparin und die Laborkontrollen während der Einstellung auf orale Antikoagulation gewissenhaft durchgeführt werden.

24 Stunden Notfallbereitschaft des behandelnden Zentrums.

Einen Sonderfall stellt zudem eine frische TVT dar, die beim immobilisierten Patienten erstdiagnostiziert wird. In diesen Fällen ist von einer Mobilisation dringend abzuraten und die Konsolidierung des Thrombus unter entsprechender Therapie und Bettruhe abzuwarten.

kurzgefasst: Die Effektivität der Kompressionstherapie nach tiefer Beinvenenthrombose ist für einen Zeitraum von 2 Jahren belegt. Die Indikation sollte im Einzelfall am Ausmaß der postthrombotischen Venenfunktion festgemacht werden. Unter bestimmten Voraussetzungen kann die Therapie der tiefen Beinvenenthrombose heute auch ambulant und unter Verzicht auf die Immobilisation des Patienten sicher und erfolgreich durchgeführt werden.

\section{Supplementåre Therapieverfahren}

Durch systemische oder lokale Lysetherapie kann eine frühe Rekanalisation der thrombosierten Venenstrombahn erreicht werden. Eine bessere Rekanalisation wäre im Hinblick auf die Verhinderung postthrombotischer Syndrome wünschenswert. Genau in diesem Punkt ist jedoch die Überlegenheit der Lysetherapie im Vergleich mit der konservativen Therapie inklusive Kompressionsstrumpf unbewiesen (20). Gut belegt hingegen ist 
das Risiko für eine lyseassoziierte Hirnblutung (ca. 1\%) und die Erhöhung der Gesamtsterblichkeit bei Lysetherapie (1-2\%). Die Durchführung einer Lysetherapie bei frischer TVT sollte daher nur noch in Ausnahmefällen diskutiert werden.

Die venöse Thrombektomie wird mit dem gleichen Therapieziel angewendet wie die Lysetherapie. Sie scheint prinzipiell geeignet für lokalisierte Thrombosen, die in deszendierender Form z.B. im Becken vorkommen können. Vergleichende Untersuchungen unter Berücksichtigung des aktuellen Standes der konservativen Therapie, die dem Anspruch moderner evidenzbasierter Medizin genügen, liegen jedoch nicht vor. Die Anwendung erscheint daher nur bei entsprechender Aufklärung darüber und über die operationsassoziierten zusätzlichen Risiken diskutabel.

Zur Verhinderung von Lungenembolien können Sperrfiltersysteme in der Vena cava inferior platziert werden. Das Problem dieser Systeme liegt darin, dass sie selbst thrombogen sind und entsprechende Folgekomplikationen verursachen können. Dies ist umso bedeutsamer als Indikationen für permanente Cavafilter das Auftreten von Lungenembolien unter Antikoagulation bzw. das Vorliegen von Kontraindikationen gegen eine therapeutische Antikoagulation sind. Der klinische Nutzen dieser Systeme konnte bis heute nicht überzeugend belegt werden (6). Als temporärer Filter kann ein Cavaschirm bei frischer emboliegefährlicher TVT z.B. perioperativ oder peripartal von Nutzen sein.

kurzgefasst: Der Nutzen supplementärer Therapieverfahren wie der Lysetherapie, der venösen Thrombektomie und der Cavaschirm-Implantation ist durch entsprechende Studien nicht belegt. Der Einsatz der genannten Verfahren ist nur in Einzelfällen unter kritischer Abwägung potenzieller Vor- und Nachteile für den Patienten und entsprechender Aufklärung zu diskutieren.

\section{Fazit}

Die Therapie der tiefen Beinvenenthrombose hat sich mit der Verfügbarkeit von niedermolekularen Heparinen vereinfacht und kann in geeigneten Fällen nun auch ambulant durchgeführt werden. Patienten mit einer relevanten Niereninsuffizienz sind jedoch für diese Therapieform ungeeignet, da niedermolekulare Heparine renal eliminiert werden und bei gestörter renaler Ausscheidungsfunktion Kumulationsgefahr mit entsprechenden Blutungsrisiken besteht. Ein weiterer Vorteil der Initialtherapie mit niedermolekularem Heparin ist der bessere Rekanalisationserfolg im Vergleich mit unfraktioniertem Heparin. Bei Kontraindikationen gegen eine orale Antikoagulation kann auch die Rezidivprophylaxe mit einer reduzierten Dosis eines niedermolekularen Heparins sicher und effektiv durchgeführt werden. Es ist jedoch zu beachten, dass die Substanzgruppe für diese Indikation derzeit nicht zugelassen ist.

Autorenerklärung: Die Autoren erklären, dass sie keine finanziellen Verbindungen mit einer Firma haben, deren Produkt in dem Artikel eine wichtige Rolle spielt (oder mit einer Firma, die ein Konkurrenzprodukt vertreibt).

\section{Literatur}

1 Agnelli G, Prandoni P, Santamaria MG et al. Three months versus one year of oral anticoagulant therapy for idiopathic deep venous thrombosis. Warfarin Optimal Duration Italian Trial Investigators. N Engl J Med 2001; 345: 165-169

2 Aschwanden M, Labs KH, Engel H et al. Acute deep vein thrombosis: early mobilization does not increase the frequency of pulmonary embolism. Thromb Haemost 2001; 85: 42-46

3 Brandjes DP, Buller HR, Heijboer $\mathrm{H}$ et al. Randomised trial of effect of compression stockings in patients with symptomatic proximal-vein thrombosis. Lancet 1997; 349: 759-762

4 Breddin HK, Hach-Wunderle V, Nakov R, Kakkar VV. Effects of a lowmolecular-weight heparin on thrombus regression and recurrent thromboembolism in patients with deep-vein thrombosis. N Engl J Med 2001; 344: 626-631

${ }^{5}$ Couturaud F, Julian JA, Kearon C. Low molecular weight heparin administered once versus twice daily in patients with venous thromboembolism: a meta-analysis. Thromb Haemost 2001; 86: 980-984

${ }^{6}$ Decousus H, Leizorovicz A, Parent F et al. A clinical trial of vena caval filters in the prevention of pulmonary embolism in patients with proximal deep-vein thrombosis. Prevention du Risque d'Embolie Pulmonaire par Interruption Cave Study Group. N Engl J Med 1998; 338: 409-415

7 DGA-Leitlinien zur Diagnostik und Therapie der Venenthrombose und Lungenembolie. VASA 2002; 31 (Suppl 60); 1-19

8 Dolovich LR, Ginsberg JS, Douketis JD et al. A meta-analysis comparing low-molecular-weight heparins with unfractionated heparin in the treatment of venous thromboembolism: examining some unanswered questions regarding location of treatment, product type, and dosing frequency. Arch Intern Med 2000; 160: 181-188

${ }^{9}$ Gonzalez-Fajardo JA, Arreba E, Castrodeza J et al. Venographic comparison of subcutaneous low-molecular weight heparin with oral anticoagulant therapy in the long-term treatment of deep venous thrombosis. J Vasc Surg 1999; 30: 283-292

${ }^{10}$ Koopman MM, Prandoni P, Piovella F et al. The Tasman Study Group. Treatment of venous thrombosis with intravenous unfractionated heparin administered in the hospital as compared with subcutaneous low-molecular-weight heparin administered at home. N Engl J Med 1996; 334: 682-687

11 Leizorovicz A, Simonneau G, Decousus $\mathrm{H}$ et al. Comparison of efficacy and safety of low molecular weight heparins and unfractionated heparin in initial treatment of deep venous thrombosis: a meta-analysis. BMJ 1994; 309: 299-304

${ }^{12}$ Lensing AW, Prins MH, Davidson BL, Hirsh J. Treatment of deep venous thrombosis with low-molecular-weight heparins: A meta-analysis. Arch Intern Med 1995; 155: 601-607

${ }^{13}$ Levine MN, Raskob G, Landefeld S, Kearon C. Hemorrhagic complications of anticoagulant treatment. Chest 2001; 119 (Suppl 1); 108S$121 \mathrm{~S}$

${ }^{14}$ Lopaciuk S, Bielska-Falda H, Noszczyk W et al. Low molecular weight heparin versus acenocoumarol in the secondary prophylaxis of deep vein thrombosis. Thromb Haemost 1999; 81: 26-31

15 Offermann M, Koepchen J, Graulich B. Die ambulante Therapie der akuten tiefen Beinvenenthrombose. Erfahrungen aus zwei Praxen für Gefäßkrankheiten in Deutschland Dtsch Med Wochenschr 2001; 126: $1164-1167$

${ }^{16}$ Schellong SM, Schwarz T, Kropp J et al. Bed rest in deep vein thrombosis and the incidence of scintigraphic pulmonary embolism. Thromb Haemost 1999; 82: 127-129

17 Schwarz T, Schmidt B, Hohlein U et al. Eligibility for home treatment of deep vein thrombosis: prospective study. BMJ 2001; 322: 12121213

${ }^{18}$ Veiga F, Escriba A, Maluenda MP et al. Low molecular weight heparin (enoxaparin) versus oral anticoagulant therapy (acenocoumarol) in the long-term treatment of deep venous thrombosis in the elderly: a randomized trial. Thromb Haemost 2000; 84: 559-564

${ }^{19}$ Warkentin TE, Levine MN, Hirsh J et al. Heparin-induced thrombocytopenia in patients treated with low-molecular-weight heparin or unfractionated heparin. N Engl J Med 1995; 332: 1330-1335

20 Wells PS, Forster AJ. Thrombolysis in deep vein thrombosis: is there still an indication? Thromb Haemost 2001; 86: 499-508 